\title{
Buoyancy-driven dispersion in confined drying of liquid binary mixtures
}

\author{
Jean-Baptiste Salmon \\ CNRS, Solvay, LOF, UMR 5258, Univ. Bordeaux, F-33600 Pessac, France. \\ Frédéric Doumenc \\ Laboratoire FAST, Univ. Paris-Sud, CNRS, Université Paris-Saclay, F-91405, Orsay, France, and \\ Sorbonne Université, UFR 919, 4 place Jussieu, F-75252, Paris Cedex 05, France.
}

(Dated: January 15, 2020)

\begin{abstract}
We investigate the impact of buoyancy on the solute mass transport in an evaporating liquid mixture (nonvolatile solute + solvent) confined in a slit perpendicular to the gravity. Solvent evaporation at one end of the slit induces a solute concentration gradient which in turn drives free convection due to the difference between the densities of the solutes and the solvent. From the complete model coupling mass transport and hydrodynamics, we first use a standard Taylor-like approach to derive a one dimensional non-linear advection-dispersion equation describing the solute concentration process for a dilute mixture. We then perform a complete analysis of the expected regimes using both scaling analysis and asymptotic solutions of this equation. The validity of this approach is confirmed using a thorough comparison with the numerical resolution of both the complete model and the 1D advection-dispersion equation. Our results show that buoyancy-driven free convection always impacts solute mass transport at long time scales, dispersing solutes in a steadily increasing length scale along the slit. Beyond this confined drying configuration, our work also provides an easy way for evaluating the relevance of buoyancy on mass transport in any other microfluidic configuration involving concentration gradients.
\end{abstract}

\section{INTRODUCTION}

Drying of liquid mixtures often leads to concentration gradients, and therefore density gradients. When these gradients are orthogonal to gravity, they inevitably generate buoyancydriven flows, that then alters the drying process when coupled to the overall mass transport. Solutal buoyancy-driven free convection is generally relevant at relatively large scales, but many recent experiments reported such flows in confined $\mathrm{mi}$ crofluidic geometries $(10-100 \mu \mathrm{m})$ : drying of confined [1-4] and sessile droplets [5-7], or any other microfluidic configuration generating concentration gradients [8, 9]. At small scales, buoyancy-driven flows are expected to play a minor role on mass transport, owing to the high viscous dissipation and fast solute diffusion [10]. Nevertheless, these flows always exist as soon as density gradients are perpendicular to gravity, and they can have an impact on less mobile species dispersed in the liquid mixture [8, 9].

The purpose of this work is to theoretically address such issues, and more precisely to quantitatively predict the range of parameters for which buoyancy-driven flows impact mass transport in a confined drying experiment. To do this, we consider the model experiment described in Fig. 11.a). A horizontal slit of height $h$, initially filled with a liquid binary mixture, is connected at one end to a tank containing the same mixture, and opened to the ambient atmosphere at the other end. This geometry is not only prone to a simple modeling, but also commonly used to probe mass transport and uni-directional drying in complex fluids ranging from colloidal dispersions to surfactant mixtures, see e.g. [11-[16]. In the following, we consider for simplicity a dilute binary mixture, solvent + non-volatile solute at concentration $\Phi$, for which both interdiffusion coefficient $D$ and kinematic viscosity $v$ are constant. Thereafter, we will also consider that the height of the slit is small enough to neglect any inertial effect. Solvent evaporation, occurring at the outlet $(X=0)$ at a rate $\dot{E}>0(\mathrm{~m} / \mathrm{s})$, leads

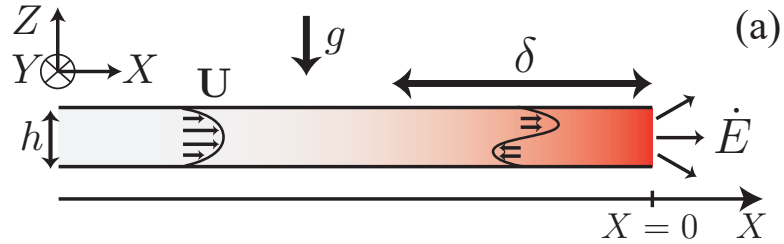

(b)

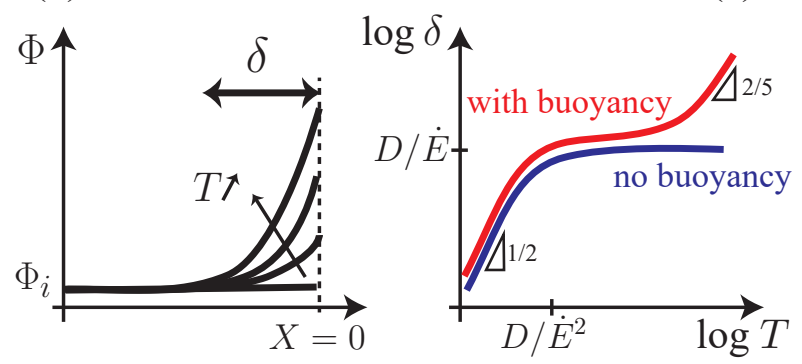

FIG. 1. (a) Schematic view of the confined drying experiment. The slit is connected at $X \rightarrow-\infty$ to a tank containing the dilute mixture at concentration $\Phi_{i}$. (b) Solvent evaporation at a rate $\dot{E}>0$ drives a flow concentrating continuously the non-volatile solute at the tip of the slit, see also the colored gradient in (a). The density gradient in turn generates a buoyancy-driven flow, superimposed on the evaporation-induced Poiseuille flow. (c) Without buoyancy, $\delta$ initially grows as $\sqrt{D T}$ before reaching a steady value $\delta \sim D / \dot{E}$, whereas the diffusive layer invades the channel as $\delta \propto T^{2 / 5}$ when buoyancy-driven free convection dominates at long time scales. These two curves are slightly shifted for the sake of clarity.

to a flow field $\mathbf{U}$ within the slit. Owing to mass conservation, the horizontal component of the fluid velocity verifies

$$
<U_{X}>=\frac{1}{h} \int_{0}^{h} U_{X} \mathrm{~d} Z=\dot{E},
$$

where $h$ is the height of the slit. This flow continuously concentrates the non-volatile solutes at the tip of the slit, in a 
layer whose typical size $\delta$ depends on the competition between evaporation-induced advection and solute diffusion, see Fig. 11(b).

Assuming first that diffusion homogenizes the concentration of solutes over $h$, the solute concentration profile $\Phi(X, T)$ obeys the following $1 \mathrm{D}$ advection-diffusion equation:

$$
\frac{\partial \Phi}{\partial T}+\dot{E} \frac{\partial \Phi}{\partial X}=D \frac{\partial^{2} \Phi}{\partial X^{2}},
$$

along with a solute no-flux boundary condition at $X=0$ as we consider non-volatile solutes. Fedorchenko and Chernov investigated theoretically this equation in the context of gas segregation induced by a moving solidification front [17] (see also Ref. [18] who used the same equation to describe stratification in drying films of colloidal dispersions). According to Ref. [17], solutes accumulate at the tip of the slit in a diffuse layer which first grows as $\delta \sim \sqrt{D T}$, and reaches the steady value $\delta \sim D / \dot{E}$ after a transient time $\sim D / \dot{E}^{2}$, see Fig. 1 (.c). In this asymptotic regime, the amount of solutes at the tip increases linearly with time, as well as the concentration gradient.

When the solute and solvent do not have the same density, such concentration gradients inevitably generate buoyancydriven free convection, see schematically Fig. 11.a). Free convection is intrinsically a multi-dimensional (2D or $3 \mathrm{D})$ problem. In the present work, we will show that free convection due to buoyancy and its consequence on solute transport in the slit sketched in Fig. 1 1 can be described by a 1D advectiondispersion equation. More precisely, we will use a standard Taylor-like approach to show that the transverse-averaged solute concentration profile defined by:

$$
\Phi_{0}(X, T)=\frac{1}{h} \int_{0}^{h} \Phi(X, Z, T) \mathrm{d} Z,
$$

is well approximated, for a wide range of parameters, by the solution of

$$
\frac{\partial \Phi_{0}}{\partial T}+\dot{E} \frac{\partial \Phi_{0}}{\partial X}=\frac{\partial}{\partial X}\left(D_{\mathrm{eff}} \frac{\partial \Phi_{0}}{\partial X}\right)
$$

where

$$
D_{\text {eff }}=D\left[1+\frac{1}{\alpha}\left(\frac{g \beta_{s} h^{4}}{v D} \frac{\partial \Phi_{0}}{\partial X}\right)^{2}\right],
$$

is a dispersion coefficient that takes into account both thermal diffusion and buoyancy on the solute mass transport. In the above equation, $g$ is the acceleration due to gravity, $\beta_{s}$ the solutal expansion coefficient of the fluid mixture, and $\alpha=$ 362880 ( $D$ is the interdiffusion coefficient and $v$ the kinematic viscosity). Chatwin and Erdogan were the first to derive this term when studying the effect of buoyancy on the dispersion of solutes in a pressure-driven flow [19]. They also reported that Taylor made the same calculation in 1953, but did not publish it, see also the review of Young and Jones on shear dispersions [20].

The physics of Eqs. (4/5) can be explained as follows. In the framework of the lubrication approximation, the density gradient induces a flow $U_{B}$ whose scale comes from a balance between buoyancy $\left(g \beta_{s} h \frac{\partial \varphi_{0}}{\partial X}\right)$ and viscous forces $\left(\nu U_{B} / h^{2}\right)$, leading to [20]:

$$
U_{B} \sim \frac{g \beta_{s} h^{3}}{v} \frac{\partial \Phi_{0}}{\partial X}
$$

This flow, and more precisely its axial velocity distribution along $X$, see Fig. 1 $1 \mathrm{a}$ ), increases solutes dispersion leading to the term in Eq. (5). This term scales as $\sim\left(U_{B} h / D\right)^{2}$ thus comparing the solute diffusive transport $(D / h)$ and its transport by buoyancy-driven convection $\left(U_{B}\right)$. The prefactor $\alpha$, as well as the power 2, comes from a standard Taylor-like perturbation approach, as first derived by Chatwin and Erdogan but in a circular tube [19]. The non-linearity of this dispersive term, compared to the Taylor-Aris dispersion in a Poiseuille flow, comes from the coupling between the buoyancy-driven flow and the density gradient: strong gradients increase the magnitude of free convection, which in turn increases solute dispersion, see Eq. (6).

Similar equations were also derived in various convection problems driven by temperature differences in a fluid layer, but also for describing the dynamics of well-mixed estuaries [21, 22], for quantifying the impact of buoyancy on the measurement of diffusivities in liquid metals [23], or even to study gravity currents of miscible fluids in porous media [24]. Despite an in-depth literature review on this classical Taylorlike approach, we are not aware of any work discussing such an equation in the context of confined drying, and more generally of microfluidic experiments generating solute gradients.

In a second step, we thus report a complete investigation of the solute concentration process described by the advectiondispersion equation Eq. (47). We first predict all the expected regimes of solute concentration using a detailed scaling analysis, and we then provide asymptotic analytical solutions of the concentration profiles when mass transport is dominated by diffusion or else by buoyancy-driven dispersion.

In particular, we show that solutes dispersion caused by buoyancy leads at long time scales to a diffusive layer thickness $\delta$ which continuously invades the channel following $\delta \propto T^{2 / 5}$, see Fig. 1(c). This theoretical approach also helps us to provide a simple diagram highlighting the range of parameters corresponding to mass transport dominated by diffusion (i.e. negligible dispersion). Finally, the validity of the $1 \mathrm{D}$ advection-dispersion model is thoroughly investigated by means of scaling analysis, and the results confirmed by comparisons with direct numerical simulations of the $2 \mathrm{D}$ model.

The present paper is organized as follows. In Section III we first present the equations modeling the experiments shown in Fig. 11. as well as the underlying assumptions. We then derive from this model the advection-dispersion equation Eq. (4). Section III then reports a complete discussion of the different regimes expected, as well as the corresponding analytical asymptotic solutions. The validity of the 1D approach is then investigated. In Section IV] we finally conclude our work by discussing its possible implications, particularly for microfluidic experiments generating concentration gradients. 


\section{FROM THE 2D MODEL TO A 1D ADVECTION-DISPERSION EQUATION}

\section{A. 2D model}

As stated in the introduction, we first consider a binary mixture, solvent + non-volatile solute with concentration $\Phi$. We also assume that its density evolves as:

$$
\rho=\rho_{i}\left[1+\beta_{s}\left(\Phi-\Phi_{i}\right)\right],
$$

where $\rho_{i}$ is the density at the solute concentration $\Phi_{i}$, and $\beta_{s}$ the solutal expansion coefficient of the fluid mixture at the reference concentration $\Phi_{i}$. The configuration under study is depicted in Fig. 11, and we consider that both the height of the slit and the evaporation-induced flow are small enough to neglect inertia. The slit is initially filled homogeneously by the solution at concentration $\Phi_{i}$. Solvent evaporation induces the concentration of the non-volatile solutes at the tip of the slit. Thereafter, we limit our study to dilute solutions, for which the evaporation rate $\dot{E}$ and the various transport coefficients (viscosity $v$, mutual diffusion coefficient $D$ ) remain constant. The following model, coupling Stokes, continuity, and solute conservation equations, is expected to describe the overall concentration process:

$$
\begin{aligned}
& \rho_{i} v \Delta \mathbf{U}-\nabla P+\left(\rho(\Phi)-\rho_{i}\right) \mathbf{g}=0, \\
& \nabla . \mathbf{U}=0, \\
& \partial_{T} \Phi+\mathbf{U} . \nabla \Phi=D \Delta \Phi,
\end{aligned}
$$

where $\mathbf{U}$ is the velocity field of the mixture, and $P$ the pressure deviation from the initial hydrostatic pressure field.

We also assume the following standard boundary conditions at the solid walls:

$$
\begin{aligned}
& \mathbf{U}=0 \text { (no slip), } \\
& \nabla \Phi . \mathbf{n}=0 \text { (no flux), }
\end{aligned}
$$

and the following standard ones at the evaporating free surface:

$$
\begin{aligned}
& U_{X}(X=0, Z, T)=\dot{E}, \\
& \left(\frac{\partial U_{Z}}{\partial X}\right)(X=0, Z, T)=0, \\
& \left(U_{X} \Phi-D \frac{\partial \Phi}{\partial X}\right)(X=0, Z, T)=0 .
\end{aligned}
$$

Eq. (15) ensures the non-volatility of the solute. At $X \rightarrow-\infty$, we impose:

$$
\Phi(X \rightarrow-\infty, Z, T)=\Phi_{i}
$$

\section{B. Numerical resolution on a given experimental case}

To illustrate the impact of buoyancy, we first consider the following realistic case: water evaporation from an aqueous dispersion of silica nanoparticles at ambient conditions in a slit of height $h=150 \mu \mathrm{m}$. Similar conditions were recently explored experimentally either to probe mass transport in such charged dispersions [14, 16, 25], or to investigate the dynamics of fractures, delamination and shear bands in the concentrated regime, see e.g. [11-13]. We will assume an initial concentration $\Phi_{i}=0.001$ and radius $a=5 \mathrm{~nm}$ for the silica nanoparticles, leading to a diffusivity $D \simeq 4.37 \times 10^{-11} \mathrm{~m}^{2} / \mathrm{s}$ according to the Stokes-Einstein relation for a temperature of $25^{\circ} \mathrm{C}$ (we do not consider here enhanced values due to colloidal interactions occurring for such systems at high concentrations [4, 16]). For such a very dilute silica dispersion, the solutal expansion coefficient at the reference concentration $\Phi_{i}$ is well approximated by $\beta_{s} \simeq \rho_{s} / \rho_{w}-1 \simeq 1.2$ where $\rho_{s} \simeq 2200 \mathrm{~kg} / \mathrm{m}^{3}$ is the density of silica, and $\rho_{w} \simeq 1000 \mathrm{~kg} / \mathrm{m}^{3}$ that of water. We assume an evaporation rate $\dot{E}=0.1 \mu \mathrm{m} / \mathrm{s}$. The latter remains constant as the volume of the colloids is much larger than the water molecular volume [26]. We will finally assume that the kinematic viscosity $v=10^{-6} \mathrm{~m}^{2} / \mathrm{s}$ remains also constant during concentration.

The numerical resolution of Eqs. $8-10$ with boundary conditions Eqs. 111 15) has been performed with the commercial software Comsol Multiphysics (finite elements, Galerkin method). The boundary condition Eq. (16) at $X \rightarrow-\infty$ has been moved to $X=-L$, where $L=10 \mathrm{~mm}$ is a finite distance large enough to not affect the results significantly (in addition, the pressure $P$ has been arbitrarily set to $P=0$ at $X=-L$ ). Time discretization is based on implicit backward differentiation formulas. Spatial discretization was achieved by a structured mesh of quadratic Lagrangian elements. The mesh convergence has been thoroughly tested by successive refinements.

Figure 2 shows the results of the numerical simulation. More precisely, Fig. 2(a) shows several snapshots at $T=10$, $10^{2}, 10^{3}, 10^{4}$, and $10^{5} \mathrm{~s}$ of both the horizontal component $U_{X}$ of the velocity field (colormap) and the concentration profile $\Phi(X, T)$ (contour). Figures 2 b) and (c) display the heightaveraged concentration profiles $\Phi_{0}$ and $U_{X}$ at $X=-300 \mu \mathrm{m}$ at the same times. For the conditions investigated, the velocity profile $U_{X}(Z, T)$ at $X=-300 \mu \mathrm{m}$ and $T=10 \mathrm{~s}$ mainly corresponds to the evaporation-driven Poiseuille flow $U_{P}=$ $6 \dot{E} Z(h-Z) / h^{2}$. However the profiles are more and more distorted at longer times by the free convection induced by the solute concentration gradient. The maximal velocity at $X=$ $-300 \mu \mathrm{m}$ due to buoyancy only, i.e. $U_{B}=U-U_{P}$, increases from $\simeq 0.24 \mu \mathrm{m} / \mathrm{s}$ at $T=10^{3} \mathrm{~s}$ to $\simeq 2 \mu \mathrm{m} / \mathrm{s}$ at $T=10^{5} \mathrm{~s}$. As clearly evidenced by the contour plot in Fig. 2(a), buoyancy also distorts the isoconcentration lines, and therefore clearly impacts the solute mass transfer. Note that this 2D model predicts that the concentration at the interface increases continuously, and the assumption of constant kinematic viscosity and mutual diffusion coefficient may not hold anymore above $T>10^{5} \mathrm{~s}$ for which the concentration at the interface reaches $\simeq 0.012$. 
(a)
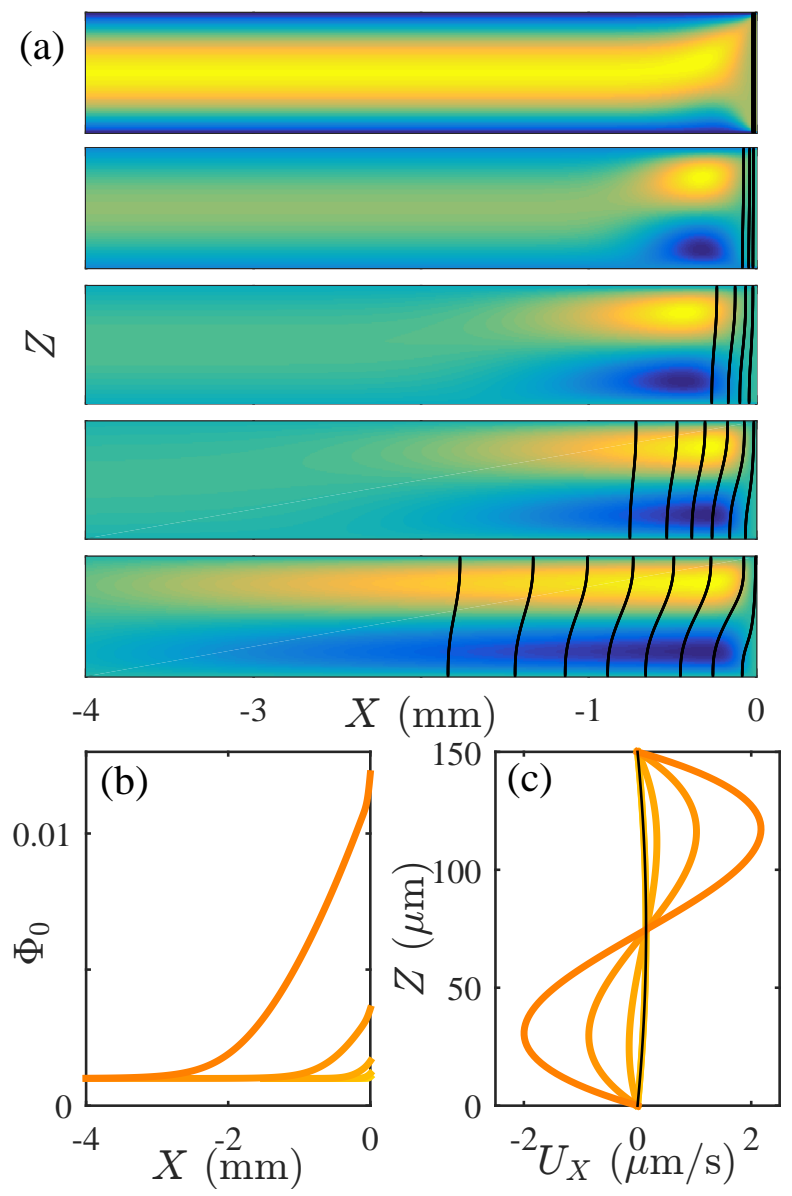

FIG. 2. (a) Snapshots of $U_{X}$ (colors) and $\Phi(X, T)$ (contours) at $T=$ $10,10^{2}, 10^{3}, 10^{4}$, and $10^{5} \mathrm{~s}$, from top to bottom. The range of the colormap is scaled by the maximal velocity in each plot (b) Heightaveraged concentration profiles $\Phi_{0}(X, T)$ and (c) velocity profiles $U_{X}(Z, T)$ at $X=-300 \mu \mathrm{m}$, at the same times $T$ as in (a). The thin dark line is the evaporation-driven Poiseuille profile $U_{P}=6 \dot{E} Z(h-$ Z) $/ h^{2}$.

\section{Advection-dispersion equation in the framework of the lubrication approximation}

As discussed in the introduction, our aim is to predict the range of parameters for which buoyancy impacts solute's transport in such confined drying configuration using a 1D advection-dispersion equation derived from the above model. As shown by Fedorchenko and Chernov [17], $D / \dot{E}$ is a length scale that naturally emerges along $X$ from the conservation equation Eq. (10), and we therefore define the following dimensionsless variables:

$$
\begin{aligned}
& z=Z / h, x=(\dot{E} / D) X, t=\left(\dot{E}^{2} / D\right) T, \\
& u_{X}=U_{X} / \dot{E}, u_{z}=U_{Z} /(\dot{E} \mathrm{Pe}), \\
& \varphi=\left(\Phi-\Phi_{i}\right) / \Phi_{i}, \quad p=h^{2} /\left(\rho_{i} v D\right) P
\end{aligned}
$$

where Pe is the Péclet number given by:

$$
\mathrm{Pe}=\frac{\dot{E} h}{D}
$$

Note that we have chosen two different scales to obtain the dimensionless coordinates $x$ and $z$. As shown later, this particular choice makes it easy to highlight the different regimes of solute concentration, while keeping a compact writing of the equations. However, it should be remembered that the scale $D / \dot{E}$ is implicitly contained in the dimensionless abscissa $x$ but not in $z$.

Using this set of dimensionless variables, one can demonstrate that the dimensionless counterpart of Eqs. 8 (10) depend only on two parameters, Pe and the solutal Rayleigh number defined as:

$$
\mathrm{Ra}=\frac{\beta_{s} \Phi_{i} g h^{3}}{v D}
$$

see Appendix A Volume integration of the continuity relation Eq. (9) yields the dimensionless counterpart of the solution global mass balance Eq. (1):

$$
<u_{x}>=\int_{0}^{1} u_{x} \mathrm{~d} z=1 .
$$

Similarly, integration of Eq. 107 yields the dimensionless solute global mass balance:

$$
\int_{x \rightarrow-\infty}^{0} \int_{z=0}^{1} \varphi \mathrm{d} z \mathrm{~d} x=t .
$$

We now assume that the typical scale $\delta$ of both the concentration gradient and the buoyancy-driven velocity field, see Fig. 11, is much larger than the channel height, i.e. $\delta \gg \mathrm{Pe}$ with our dimensionless variables, Eqs. (17). We therefore assume quasi-parallel flows and we use the standard lubrication approximation [27] to derive a 1D solute conservation equation from the above model. More precisely, we use a standard Taylor-like perturbation method, as reviewed for instance in Young and Jones' work on shear dispersion [20], and expand the concentration field as:

$$
\varphi(x, z, t)=\varphi_{0}(x, t)+\operatorname{Pe}^{2} \varphi_{1}(x, z, t),
$$

where $\varphi_{0}(x, t)$ is the transverse-averaged concentration profile, i.e. $\varphi_{0}(x, t)=\langle\varphi(x, z, t)>$ with the same averaging as in Eq. 20, and $\operatorname{Pe}^{2} \varphi_{1} \ll \varphi_{0}$. Appendix A presents the detailed derivation leading ultimately to the following transport equation for the mean concentration field $\varphi_{0}$ :

$$
\frac{\partial \varphi_{0}}{\partial t}+\frac{\partial \varphi_{0}}{\partial x}=\frac{\partial}{\partial x}\left(D_{\mathrm{eff}} \frac{\partial \varphi_{0}}{\partial x}\right),
$$

assuming $t \gg \mathrm{Pe}^{2}, \delta \gg \mathrm{Pe}$, and $\mathrm{Pe}^{2} \varphi_{1} \ll \varphi_{0}$ [20]. The range of validity of these conditions will be discussed later on in Sec.IIID.

The dispersion coefficient $D_{\text {eff }}$ is given by:

$$
\begin{aligned}
& D_{\text {eff }}=1+\frac{(\mathrm{PeRa})^{2}}{\alpha}\left(\frac{\partial \varphi_{0}}{\partial x}\right)^{2}+\beta \mathrm{Pe}^{2} \\
& \simeq 1+\frac{(\mathrm{PeRa})^{2}}{\alpha}\left(\frac{\partial \varphi_{0}}{\partial x}\right)^{2}
\end{aligned}
$$


where $\beta=1 / 210$ and $\alpha=362880$. The first term corresponds to the dispersion of the solutes due to the axial velocity distribution caused by the density gradient, as discussed in Introduction, whereas the second is the traditional Taylor-Aris term induced by the Poiseuille flow. There are no coupling terms in the geometry under study, between buoyancy and Taylor-Aris dispersion, owing to the symmetry along the plane $z=1 / 2$, as already noted in [20]. Eqs. 232 24) finally lead to Eqs. (4) 5) with real units as we will assume that the Taylor-Aris term is negligible in our configuration, i.e. $\beta \mathrm{Pe}^{2} \ll 1$, see Sec. III D regarding the range of validity of our model. Note importantly that despite the fact that the dispersion term due to buoyancy in Eq. 24] depends explicitly on the Péclet number Pe in our dimensionless model, the latter actually does not depend on the evaporation rate $\dot{E}$ due to the scale $D / \dot{E}$ used to define $x$, see Eqs. (17).

It should also be noted that many groups recently investigated the drying of droplets confined between two circular parallel plates, see e.g. [1-4, 9]. Most of these works clearly reported buoyancy-driven flows generated by the radial density gradients induced by solvent evaporation. Interestingly, the same above calculations applied to this cylindrical geometry and for a binary mixture, lead also to Eqs. 23 24] with $\alpha=362880$ but without the Taylor-Aris and advection terms and with cylindrical coordinates. Our theoretical derivation does therefore not only apply to the case of a slit, but also to this specific $2 \mathrm{D}$ configuration.

\section{DYNAMICS OF THE SOLUTE CONCENTRATION}

In the following, we now turn to a thorough analysis of the solute concentration process in the confined drying experiment described in Fig. 1. More precisely, we will use the $1 \mathrm{D}$ advection-dispersion equation derived above, Eq. [23, to unveil both the expected regimes of solute concentration and the relevant parameters impacting the solute mass transport. Eq. 23) is supplemented with the following initial and boundary conditions:

$$
\begin{aligned}
& \varphi_{0}(x, t=0)=0, \\
& \lim _{x \rightarrow-\infty} \varphi_{0}(x, t)=0, \\
& \left(1+\varphi_{0}-D_{\text {eff }} \frac{\partial \varphi_{0}}{\partial x}\right)(x=0, t),
\end{aligned}
$$

where Eq. 27) ensures the condition of zero solute flux through the free surface (non volatility of the solute).

In the framework of this 1D model, the global solute mass balance Eq. 21 reads:

$$
\int_{x \rightarrow-\infty}^{0} \varphi_{0}(x, t) \mathrm{d} x=t .
$$

Finally, an important feature of the concentration field is its spatial extent $\delta$ as illustrated in Fig. 1. and we define the latter according to:

$$
\delta(t)=-\frac{\int_{-\infty}^{0} x \varphi_{0}(x, t) \mathrm{d} x}{\int_{-\infty}^{0} \varphi_{0}(x, t) \mathrm{d} x}=-\frac{1}{t} \int_{-\infty}^{0} x \varphi_{0}(x, t) \mathrm{d} x .
$$

\section{A. Scaling analysis}

We first use a standard method [28] to derive the scaling laws of the model defined by the governing equation Eq. (23), along with the initial and boundary conditions Eq. 25 27 . The global mass balance Eq. (28), which implicitly contains the governing equation and its boundary and initial conditions, provides a first scaling law:

$$
\varphi_{0} \delta \sim t
$$

A second scaling law is provided by Eq. 23), which can be written as a relation between 4 positive terms:

$$
\frac{\partial \varphi_{0}}{\partial t}+\frac{\partial \varphi_{0}}{\partial x}=\frac{\partial^{2} \varphi_{0}}{\partial x^{2}}+\frac{(\mathrm{PeRa})^{2}}{\alpha} \frac{\partial}{\partial x}\left(\frac{\partial \varphi_{0}}{\partial x}\right)^{3} .
$$

Owing to the boundary condition Eq. 26], the order of magnitude of these four terms is:

$$
\frac{\varphi_{0}}{t} ; \frac{\varphi_{0}}{\delta} ; \frac{\varphi_{0}}{\delta^{2}} ; \quad \frac{(\mathrm{PeRa})^{2}}{\alpha} \frac{\varphi_{0}^{3}}{\delta^{4}}
$$

As time goes by, different regimes are encountered depending on what couple of terms dominates in Eq. (31). As all the terms of this equation are positive, the balance of the different orders of magnitude reads:

$$
\max \left(\frac{\varphi_{0}}{t}, \frac{\varphi_{0}}{\delta}\right) \sim \max \left(\frac{\varphi_{0}}{\delta^{2}}, \frac{(\mathrm{PeRa})^{2}}{\alpha} \frac{\varphi_{0}^{3}}{\delta^{4}}\right),
$$

and one therefore expects four different regimes, systematically reviewed in the following.

a. Diffusive regime $D 1-$ We define this regime as the one corresponding to $\frac{\varphi_{0}}{t} \sim \frac{\varphi_{0}}{\delta^{2}}$ in Eq. 33. Combining this relation with Eq. 30] yields:

$$
\begin{aligned}
& \delta \sim \sqrt{t} \text { and } \varphi_{0} \sim \sqrt{t}, \\
& t \ll 1 \text { and } \frac{\mathrm{PeRa}}{\sqrt{\alpha}} \ll 1,
\end{aligned}
$$

b. Diffusive regime $D 2-$ This regime corresponds to $\frac{\varphi_{0}}{\delta} \sim \frac{\varphi_{0}}{\delta^{2}}$ and thus to

$$
\begin{aligned}
& \delta \sim 1 \text { and } \varphi_{0} \sim t, \\
& t \gg 1 \text { and } t \ll\left(\frac{\mathrm{PeRa}}{\sqrt{\alpha}}\right)^{-1},
\end{aligned}
$$

and D2 can thus only be observed when PeRa $\ll \sqrt{\alpha}$

c. Dispersive regime $\mathrm{Cl}-$ This regime corresponds to $\frac{\varphi_{0}}{t} \sim \frac{(\mathrm{PeRa})^{2}}{\alpha} \frac{\varphi_{0}^{3}}{\delta^{4}}$, and after calculation to

$$
\begin{aligned}
& \delta \sim\left(\frac{\mathrm{PeRa}}{\sqrt{\alpha}}\right)^{1 / 3} \sqrt{t} \text { and } \varphi_{0} \sim\left(\frac{\mathrm{PeRa}}{\sqrt{\alpha}}\right)^{-1 / 3} \sqrt{t}, \\
& t \ll\left(\frac{\mathrm{PeRa}}{\sqrt{\alpha}}\right)^{2 / 3} \text { and }\left(\frac{\mathrm{PeRa}}{\sqrt{\alpha}}\right) \gg 1
\end{aligned}
$$


d. Dispersive regime $\mathrm{C} 2-$ This last regime corresponds to $\frac{\varphi_{0}}{\delta} \sim \frac{(\mathrm{PeRa})^{2}}{\alpha} \frac{\varphi_{0}^{3}}{\delta^{4}}$, leading after calculation to

$$
\begin{aligned}
& \delta \sim\left(\frac{\mathrm{PeRa}}{\sqrt{\alpha}}\right)^{2 / 5} t^{2 / 5} \text { and } \varphi_{0} \sim\left(\frac{\mathrm{PeRa}}{\sqrt{\alpha}}\right)^{-2 / 5} t^{3 / 5}, \\
& t \gg\left(\frac{\mathrm{PeRa}}{\sqrt{\alpha}}\right)^{2 / 3} \text { and } t \gg\left(\frac{\mathrm{PeRa}}{\sqrt{\alpha}}\right)^{-1} .
\end{aligned}
$$

The above scaling analysis reveals the importance of the dimensionless parameter PeRa. When PeRa $\ll \sqrt{\alpha}$, the sequence of regimes is $\mathrm{D} 1 \rightarrow \mathrm{D} 2 \rightarrow \mathrm{C} 2$ with two transition times $t_{\mathrm{D} 1 \rightarrow \mathrm{D} 2} \sim 1$ and

$$
t_{\mathrm{D} 2 \rightarrow \mathrm{C} 2} \sim\left(\frac{\mathrm{PeRa}}{\sqrt{\alpha}}\right)^{-1} .
$$

For this range of PeRa, we found at small time scales the classical scenario of Fedorchenko and Chernov [17] expected without buoyancy and displayed in Fig. 1.(c): the square-root growth $\delta \sim \sqrt{t}(\delta \sim \sqrt{D T}$ with real units) of a diffusive layer reaching after a transient $t \sim 1\left(T \sim D / \dot{E}^{2}\right)$, a constant value $\delta \sim 1(\delta \sim D / \dot{E})$ owing to the competition between solute diffusion and evaporation-driven advection. On longer time scales, however, the solute concentration gradient steadily increases, and buoyancy can no longer be ignored (transition D2 $\rightarrow \mathrm{C} 2$ ).

When PeRa $\gg \sqrt{\alpha}$, dispersion caused by buoyancy always dominates diffusion in the solute mass transport, and the sequence of observed regimes reduces to $\mathrm{C} 1 \rightarrow \mathrm{C} 2$ with a transition at $t_{\mathrm{C} 1 \rightarrow \mathrm{C} 2} \sim(\mathrm{PeRa} / \sqrt{\alpha})^{2 / 3}$. Importantly, this scaling analysis also demonstrates that solutes are always dispersed at long time scales in a steadily increasing diffusive layer following $\delta \propto t^{2 / 5}$.

\section{B. Numerical simulation of the $1 D$ advection-dispersion model and asymptotic solutions}

To illustrate the above scaling analysis, Fig. 33(a) displays the temporal evolution of the thickness of the diffusive layer $\delta$, calculated from the numerical resolution of Eqs. (23,277) for a wide range of PeRa ranging logarithmically from $10^{-2}$ to $10^{4}$.

Except for PeRa $\geq \sqrt{\alpha}$, these curves display three regimes: an initial growth of the diffusive layer following $\delta \sim \sqrt{t}$ (regime D1), a constant plateau $\delta \simeq 1$ reached at $t \simeq 1$ (regime D2), followed again by the growth of $\delta$ at longer time scales according to $\delta \propto t^{2 / 5}$ (regime $\mathrm{C} 2$ ). The departure from the regime $\mathrm{D} 2$ to the regime $\mathrm{C} 2$ occurs at a critical time which decreases for increasing PeRa. For PeRa $\geq \sqrt{\alpha}$, the regime D2 of constant diffusive layer does not exist, and the initial regime $\mathrm{C} 1$ for which we observe again $\delta \propto \sqrt{t}$, does not collapse with the other curves in the regime D1. Figures 3 (b-d) illustrate the specific case $\mathrm{PeRa}=1$, for which the sequence of the three different regimes D1 $\rightarrow \mathrm{D} 2 \rightarrow \mathrm{C} 2$ is easily revealed.

All these numerical results are obviously in line with the scaling analysis reported in Sec. III A, but one should go beyond the scaling laws Eqs. 34 41) in order to predict quan-
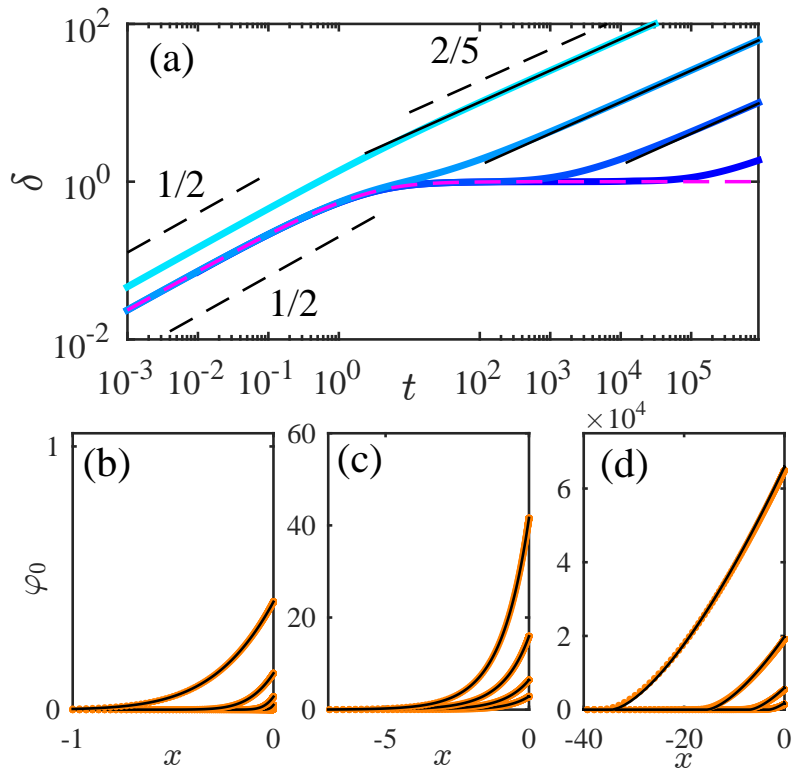

FIG. 3. (a) Numerical solution $\delta$ vs. $t$ of the $1 \mathrm{D}$ advection-dispersion model Eqs 23 27) for different PeRa: $10^{-2}, 10^{0}, 10^{2}$, and $10^{4}$ (from dark to light blue). The magenta dashed line is the theoretical prediction Eq. 44) in the diffusive regimes D1 and D2. The black lines are the approximations Eq. 45 for the dispersive regime C2. (b) to (d): $\varphi(x, t)$ vs. $x$ computed from Eqs 23 27) at PeRa $=1$. (b) regime D1: $t<0.5$; (c) D2: $1<t<100$; and (d) C2: $t>1000$. In (b) and (c), the analytical solutions given by Eq. B1 (black lines) are superimposed with the numerical resolution. In (d), black lines correspond to the approximate solution Eq. C5.

titatively the range of parameters for which the solute mass transport is dominated by diffusion only.

When diffusion dominates mass transport (regimes D1 and D2), Eq. 23 reduces to the following linear advectiondiffusion equation:

$$
\frac{\partial \varphi_{0}}{\partial t}+\frac{\partial \varphi_{0}}{\partial x}=\frac{\partial^{2} \varphi_{0}}{\partial x^{2}}
$$

The analytical solution of this advection-diffusion equation has been derived by Fedorchenko and Chernov [17] see Eq. (B1) in Appendix B. This analytical solution superimposes perfectly on the profiles computed numerically from Eq. 23) at small time scales, see Fig. 3(b) and (c). We also computed $\delta$ vs. $t$ from this analytical solution:

$$
\begin{aligned}
\delta(t)=\frac{-t^{2}+[t(4+t)-4] \operatorname{erf}\left(\frac{\sqrt{t}}{2}\right)}{4 t} \\
+\frac{(2+t) \sqrt{t} \exp \left(-\frac{t}{4}\right)}{2 \sqrt{\pi} t},
\end{aligned}
$$

see the magenta dashed line in Fig. 3 (a).

In the dispersive regime $\mathrm{C} 2$, we used the integral method [29] to approximate the concentration profiles. The detailed calculation and the resulting approximations are 
given in Appendix C see in particular Eq. C5 from which we computed $\delta(t)$ :

$$
\delta(t)=\frac{5^{2 / 5} 3^{3 / 5}}{7}\left(\frac{\mathrm{PeRa}}{\sqrt{\alpha}} t\right)^{2 / 5} .
$$

Both $\delta$ vs. $t$ and concentration profiles perfectly superimpose on the solutions computed numerically, see Fig. 3 (a) and Fig. 3(d) respectively.

We did not find an approximate solution of the concentration profiles in the dispersive regime $\mathrm{C} 1$. Nevertheless, we computed numerically the prefactor in Eq. (38) leading to:

$$
\delta(t) \simeq 0.55\left(\frac{\mathrm{PeRa}}{\sqrt{\alpha}}\right)^{1 / 3} \sqrt{t} .
$$

\section{Transition times}

We define a criterion for evaluating quantitatively the critical time corresponding to the transition from a diffusive regime, for which buoyancy-driven dispersion exists but has a negligible impact on the solute transport, to a dispersive regime, for which buoyancy significantly disperses solutes along the slit. We consider that this transition occurs when the ratio of the dispersion flux to the height-averaged diffusive flux reaches a given value $p$. This condition reads:

$$
\frac{<u_{x} \varphi>-\varphi_{0}}{\frac{\partial \varphi_{0}}{\partial x}}=p
$$

at a given abscissa $x$. We arbitrarily set $p=0.2$ for all the calculations in the present work. In the framework of the 1D advection-dispersion model, Eq. (47) turns to

$$
D_{\text {eff }}=1+p .
$$

Using the expression of the dispersion coefficient Eq. 24) and considering that the concentration gradient is maximum at $x=$ 0 , Eq. (48) reads

$$
\operatorname{PeRa}\left(\frac{\partial \varphi_{0}}{\partial x}\right)_{x=0, t=t_{\mathrm{D} 2 \rightarrow \mathrm{C} 2}}=\sqrt{p \alpha}
$$

The critical time $t_{\mathrm{D} 2 \rightarrow \mathrm{C} 2}$ is computed by numerically solving Eq. (49), where the concentration gradient at $x=0$ is estimated from the analytical expression Eq. (B2), valid in the diffusive regimes D1 and D2. This results in the diagram shown in Fig. 4, evidencing the transition from the diffusiondominated regimes D1 and D2 towards the dispersive regime (C2).

The 1D advection-dispersion model predicts that for $\mathrm{PeRa} \geq \sqrt{p \alpha}$, buoyancy always dominates solute transport, regardless of the time (regimes $\mathrm{C} 1$ and $\mathrm{C} 2$ ). In this range of parameters, we estimated the transition time $t_{\mathrm{C} 1 \rightarrow \mathrm{C} 2}$ from the cross-over of $\delta$ vs. $t$ in both regimes $\mathrm{C} 1$ and $\mathrm{C} 2$, see Eq. (45) and Eq. [46, leading to:

$$
t_{\mathrm{C} 1 \rightarrow \mathrm{C} 2} \simeq 0.6\left(\frac{\mathrm{PeRa}}{\sqrt{\alpha}}\right)^{2 / 3} .
$$

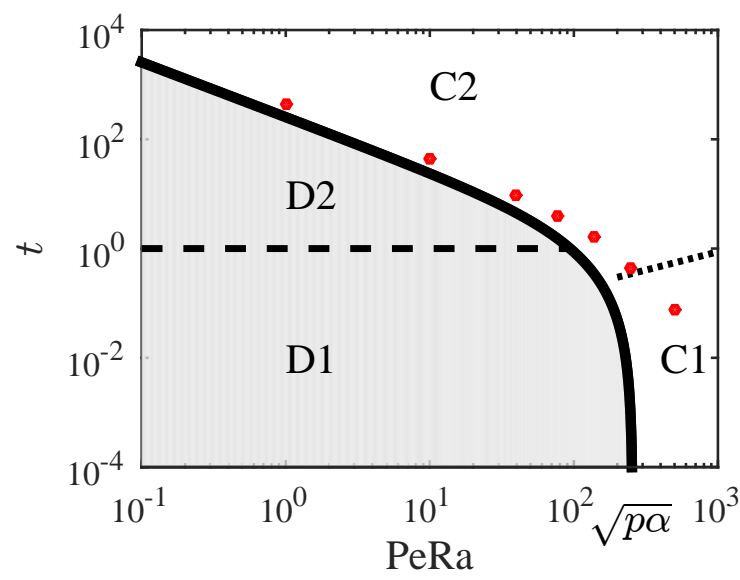

FIG. 4. Diagram highlighting the different regimes of solute mass transport predicted by the $1 \mathrm{D}$ advection-dispersion model, Eqs. 23 . 27). The thick line corresponds to $t_{\mathrm{D} 2 \rightarrow \mathrm{C} 2}$ estimated from Eqs. 49 . B2). The shaded area corresponds to the diffusive regimes D1 and D2. The dotted line corresponds to the transition between the dispersive regimes $\mathrm{C} 1 \rightarrow \mathrm{C} 2$, estimated from Eq. 50). The red bullets represent the transition from a diffusive to a dispersive regime, estimated with the $2 \mathrm{D}$ model, Eqs. $\mathrm{A} 1 \mathrm{~A} 8$, for $\mathrm{Pe} \simeq 0.252$ and $\mathrm{Ra}$ varying from 4 to 2000 (critical time estimated from Eq. 47)).

Figure 4 summarizes all these results and can therefore serve as a guide to predict the expected transport regimes in a given experimental configuration using a single dimensionless parameter PeRa.

\section{Validity and limitations of the 1D advection-dispersion model}

The 1D advection-dispersion model is based on several simplifying assumptions, whose validity must be carefully checked in order to validate the results in a given configuration.

The assumptions required to validate Eq. A11, on which the estimation of the dispersion term in Eq. A9 is based, are the following ones (see Appendix A:

$$
\begin{aligned}
& t \gg \mathrm{Pe}^{2}, \\
& \delta \gg \mathrm{Pe}, \\
& \operatorname{Pe}^{2} \varphi_{1} \ll \varphi_{0} .
\end{aligned}
$$

The last inequality Eq. (53) demands that the transverse dispersion time $\delta^{2} / D_{\text {eff }}$ remains longer than the diffusion time across the channel height $\sim \mathrm{Pe}^{2}[20]$. In addition, $\beta \mathrm{Pe}^{2} \ll 1$ is required to neglect the Taylor-Aris contribution in Eq. (24). As $\beta=1 / 210(1 / \sqrt{\beta} \simeq 14.5)$, this last assumption is always verified for $\mathrm{Pe} \lesssim 1$ (i.e. $\mathrm{Pe} \ll 1$ or $\mathrm{Pe} \sim 1$ ). In the remainder of this analysis, we will show that $\mathrm{Pe} \lesssim 1$ results in the validity of conditions Eqs. (51 53) from $t=0$ to $t \rightarrow \infty$ when $\mathrm{PeRa} \ll \sqrt{\alpha}$ (asymptotic case 1 in the following analysis), and from $t \gg \mathrm{Pe}^{2}$ to $t \rightarrow \infty$ when PeRa $\gg \sqrt{\alpha}$ (asymptotic case 2). 
a. Case 1: $\mathrm{PeRa} / \sqrt{\alpha} \ll 1$, corresponding to the succession of regimes D1, D2, C2, with a transition from the diffusive regime D2 to the dispersive regime C2 at time $t_{\mathrm{D} 2 \rightarrow \mathrm{C} 2}$ given by Eq. (42). We show in appendix $\mathrm{D}$ that conditions Eqs. (51, 53) are always satisfied for times $t \gg t_{\mathrm{D} 2 \rightarrow \mathrm{C} 2}$. Therefore, the model is expected to describe correctly the dispersive regime C2. Conditions Eqs. 51 53 might be wrong in regimes D1 or D2 (i.e. for $t \ll t_{\mathrm{D} 2 \rightarrow \mathrm{C} 2}$ ). But no significant loss of accuracy is expected, since buoyancy-driven dispersion is negligible in these diffusive regimes. Consequently, the 1D advection-dispersion model is valid at all times, from $t=0$ to $t \rightarrow \infty$. This is illustrated in Fig. 5. where the 1D advectiondispersion model is compared with the output of the 2D model defined by Eqs. A1 A8, for $\mathrm{Pe} \simeq 0.344$ and $\mathrm{Ra} \simeq 910$, leading to $\mathrm{PeRa} / \sqrt{\alpha} \simeq 0.52$. The agreement is almost perfect for both $\varphi_{0}$ and $\delta$, from short to long times. An exception is the weak loss of accuracy of the 1D advection-dispersion model for the estimation of $\varphi_{0}$ close to the boundary at $x=0$ (see the inset in Fig. 5a), because the lubrication theory does not fit the boundary condition Eq. A5. As expected, the discrepancy between both models is limited to a region whose extent is of the order of Pe (the height of the slit $h$ scaled by the reference horizontal length $D / \dot{E}$ ).

b. Case 2: $\mathrm{PeRa} / \sqrt{\alpha} \gg 1$, corresponding to the succession of the dispersive regimes $\mathrm{C} 1$ and $\mathrm{C} 2$. We show in Appendix D that Eqs. 51 53 are all verified as soon as $t \gg \mathrm{Pe}^{2}$. The 1D advection-dispersion model must therefore be used carefully in this case, because this condition is not valid at the beginning, for short times since $\mathrm{Pe} \lesssim 1$. This point is illustrated in Fig. 4. where the transition from the diffusive to the dispersive regimes has been determined with the 1D advection-dispersion model (thick continuous line) and with the 2D model defined by Eqs. A1 A8) (red bullets). In the latter case, we set $\mathrm{Pe} \simeq 0.252$ and $\mathrm{Ra}$ has been varied from 4 to 2000. The critical times estimated with the 2D model are close to the estimates given by the $1 \mathrm{D}$ advection-dispersion model, except for the two highest PeRa, corresponding to the succession of the $\mathrm{C} 1-\mathrm{C} 2$ regimes in the 1D approach. The 2D model shows that a diffusive regime always exists at small times, whereas the 1D model, which is not valid at short times $t \lesssim \mathrm{Pe}^{2}$, erroneously predicts that the dispersive regime begins from $t=0$.

\section{CONCLUSION AND DISCUSSIONS}

In the present work, we investigated the role of buoyancydriven free convection on the solute mass transport in a model experimental configuration: solvent evaporation from a dilute mixture confined in a horizontal slit. To quantify the impact of buoyancy, we derived a 1D advection-dispersion equation as traditionally done in the context of shear dispersion. This equation displays a dispersion coefficient $D_{\text {eff }}$ to account for buoyancy-driven flows, see Eq. (5). Solute mass transport remains dominated by diffusion as long as:

$$
\left(\frac{g \beta_{s} h^{4}}{v D} \frac{\partial \Phi_{0}}{\partial X}\right)^{2} \ll \alpha
$$

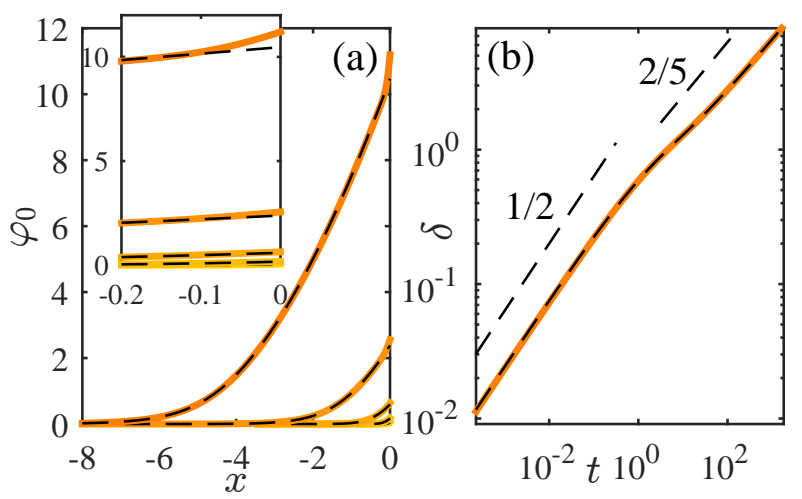

FIG. 5. (a) Average dimensionless concentration profiles $\varphi_{0}(x, t)$ for both the 2D model (same parameters as in Sec II Bleading to $\mathrm{Pe} \simeq$ 0.344 and $\mathrm{Ra} \simeq 910$ ), and the solution of the advection-dispersion equation Eq. (23) for PeRa $\simeq 313$ (black dotted lines), at times $t \simeq$ $2.29 \times 10^{-3}, 2.29 \times 10^{-2}, 0.229,2.29$ and 22.9. The inset shows a zoom close to the drying interface $x=0$. (b) Extent of the diffusive layer $\delta$ defined by Eq. 29) for the 2D model and the 1D model (black dotted line) with the same parameters as in (a).

with real units, where $\alpha=362880$. We then performed a complete analysis of the expected regimes in the configuration shown in Fig. 11, and we showed in particular that the solutes transport is always dominated by buoyancy-driven dispersion at long time scales leading to a continuously increasing diffusive layer as $\delta \propto T^{2 / 5}$. A critical point of our model is the assumption of constant mutual diffusion coefficient and viscosity during the solute concentration, and observing the different regimes predicted above maybe challenging in a single experiment (see for instance the case explored in Fig. 2 and the associated discussion). Eq. (5) can be easily modified to include a slowing down of the evaporation rate and a nonconstant mutual diffusion coefficient at high solute concentration, as done for instance in Ref. [30] in a similar configuration for polymer and surfactant solutions, but without buoyancy. Nevertheless, the different regimes predicted above, and their associated scaling laws, could be impacted by these effects.

Note also that our work focused on the case of a slit, and we included in the above calculation the dispersion due to the Poiseuille flow, see the Taylor-Aris term in Eq. (24). Moreover, there are no coupling terms between buoyancy and Taylor-Aris dispersion owing to the symmetry of the geometry investigated, and we had therefore neglected the dispersion due to the Poiseuille flow. Interestingly, the case of a capillary tube with a finite width (as a square or a circular cross-section) should deserve further attention. Indeed, a calculation similar to that performed above for such geometries can be carried out, but density gradients in the transverse dimension lead to transverse flows that couple to the longitudinal dispersion. To our knowledge, Chatwin and Erdogan [19] were the first to mention this subtle point when studying the dispersion of a solute flowing in a straight circular tube in the presence of buoyancy. They even showed that these transverse flows could decrease the buoyancy-induced longitudinal dispersion for a given range of parameters. For capillary tubes, 
one cannot also exclude (possibly non-negligible) coupling terms between Taylor-Aris and buoyancy-driven dispersions in the configuration shown in Fig. 1. We hope in a near future to investigate these subtle issues in detail.

Beyond the specific configuration investigated in the present work, different microfluidic tools have recently been developed to measure accurately the mutual diffusion coefficient of liquid mixtures owing to the very precise control of experimental conditions and mass transport at small scales [31-33]. In this context, Eq. (5] makes it possible to rigorously estimate the impact of buoyancy on such measurements. More specifically, experiments exploiting drying to induce concentration gradients, similarly to the experiment shown in Fig. 1, were even recently reported to measure mutual diffusion coefficients of various complex fluids, namely copolymer solutions [1] and charged colloidal dispersions [4, 16]. For the latter case, the rheological properties of the colloidal dispersions strongly evolve with the colloid (solute) concentration up to reaching the formation of colloidal glasses at a concentration below the colloid closepacking. Nevertheless, buoyancy-driven flows were clearly evidenced in the liquid regime [4], and rough estimates using Eq. (54) clearly indicate that buoyancy plays an important role for mass transport, casting some doubts on the coefficient values reported in [4] at low colloid concentrations.

Beyond these measurements of mutual diffusion coefficients, our work may be also of interest to evaluate the impact of buoyancy-driven dispersion for any other microfluidic configuration generating concentration gradients. In particular, many recent works focused on diffusio-phoresis, i.e. the transport of colloidal particles induced by solute concentration gradients [34-36]. The role of buoyancy in such experiments was even investigated recently in detail by $\mathrm{Gu}$ et al. [8]. Using scaling arguments, they identified conditions for which buoyancy negligibly impacts solute mass transport, leading to an inequality similar to Eq. (54) but with a significantly different numerical constant $\left(96^{2}=9216\right.$ instead of $\alpha=362880$ for a slit). Our theoretical development based on a standard pertubative approach [20] may therefore help to refine the range of parameters for which buoyancy-driven dispersion does not play any role in such experiments. Diffusio-phoretic effects are moreover expected to play a crucial role in evaporating liquid mixtures of colloids of different sizes, possibly leading to stratified materials [18]. We also hope that our simple model of uni-directional drying may be relevant to evaluate the role of buoyancy in similar configurations, in particular when gradients are orthogonal to gravity as in evaporationinduced propagating fronts in drying liquid films [37].

\section{ACKNOWLEDGMENTS}

We thank Solvay and CNRS for fundings.

\section{Appendix A: Derivation of the advection-dispersion equation}

Using the dimensionless variables given by Eqs. (17), Eqs. 8,10, read:

$$
\begin{aligned}
& \left(\operatorname{Pe}^{2} \frac{\partial^{2}}{\partial x^{2}}+\frac{\partial^{2}}{\partial z^{2}}\right) u_{x}=\frac{\partial p}{\partial x}, \\
& \operatorname{Pe}^{2}\left(\operatorname{Pe}^{2} \frac{\partial^{2}}{\partial x^{2}}+\frac{\partial^{2}}{\partial z^{2}}\right) u_{z}=\frac{\partial p}{\partial z}+\operatorname{Ra} \varphi, \\
& \nabla \cdot \mathbf{u}=0, \\
& \frac{\partial \varphi}{\partial t}+\mathbf{u} \cdot \nabla \varphi=\left(\frac{\partial^{2}}{\partial x^{2}}+\frac{1}{\operatorname{Pe}^{2}} \frac{\partial^{2}}{\partial z^{2}}\right) \varphi,
\end{aligned}
$$

The dimensionless initial condition is $\varphi(x, z, t=0)=0$ and the dimensionless boundary conditions are:

$$
\begin{aligned}
& u_{x}(x=0, z, t)=1, \\
& \left(\frac{\partial u_{z}}{\partial x}\right)(x=0, z, t)=0, \\
& \left(1+\varphi-\frac{\partial \varphi}{\partial x}\right)(x=0, z, t)=0, \\
& \varphi(x \rightarrow-\infty, z, t)=0
\end{aligned}
$$

along with the dimensionless counterpart of Eqs. (11|12) at the solid walls.

Averaging the transport equation $\mathrm{A} 4$ over the height $h$ leads to, with the help of Eq. (22)

$$
\frac{\partial \varphi_{0}}{\partial t}+\frac{\partial \varphi_{0}}{\partial x}+\operatorname{Pe}^{2} \frac{\partial<u_{x} \varphi_{1}>}{\partial x}=\frac{\partial^{2} \varphi_{0}}{\partial x^{2}} .
$$

Subtracting this last relation to Eq. A4 results in:

$$
\begin{array}{r}
\frac{\partial \varphi_{1}}{\partial t}+\frac{\left(u_{x}-1\right)}{\mathrm{Pe}^{2}} \frac{\partial \varphi_{0}}{\partial x}+\mathbf{u} \cdot \nabla \varphi_{1}-\frac{\partial<u_{x} \varphi_{1}>}{\partial x}= \\
\frac{\partial^{2} \varphi_{1}}{\partial x^{2}}+\frac{1}{\operatorname{Pe}^{2}} \frac{\partial^{2} \varphi_{1}}{\partial z^{2}} .
\end{array}
$$

The continuity equation Eq. A3 imposes the scaling $u_{z} \sim$ $u_{x} / \delta$, and Eq. A10 therefore leads to:

$$
\frac{\partial^{2} \varphi_{1}}{\partial z^{2}} \simeq\left(u_{x}-1\right) \frac{\partial \varphi_{0}}{\partial x} .
$$

assuming $t \gg \mathrm{Pe}^{2}, \delta \gg \mathrm{Pe}$, and $\mathrm{Pe}^{2} \varphi_{1} \ll \varphi_{0}$ [20].

Similarly, the leading-order terms in the Stokes equation Eqs. A1 A2 lead to the horizontal component of the velocity field:

$$
\begin{aligned}
& u_{x}(x, z, t)=u_{x}^{P}(z)+u_{x}^{B}(x, z, t), \\
& u_{x}^{P}(z)=6 z(1-z), \\
& u_{x}^{B}(x, z, t)=-\frac{\operatorname{Ra}}{12} \frac{\partial \varphi_{0}}{\partial x} z(2 z-1)(z-1),
\end{aligned}
$$

checking both the global mass balance Eq. 20, and the no-slip boundary conditions on the solid walls. The term $u_{x}^{P}$ is simply the Poiseuille flow induced by solvent evaporation, whereas the second term $u_{x}^{B}$, known as the Birikh profile [38], corresponds to the flow induced by buoyancy, see Fig.11.a). Notice 
that the velocity field Eq. A12 does not fit the boundary condition Eq. (A5). This is a usual drawback of the lubrication theory which results in a loss of accuracy in the vicinity of the interface at $x=0$, see Sec. IIID for a discussion.

$\varphi_{1}$ can now be evaluated from Eq. A11, assuming the noflux boundary condition at $z=0$ and $z=1$, and imposing $<$ $\varphi_{1}>=0$. Using the linearity of Eq. A11, we look separately for the solutions $\varphi_{1}^{P}$ and $\varphi_{1}^{B}$ due to the Poiseuille flow and buoyancy respectively, i.e.:

$$
\begin{aligned}
\frac{\partial^{2} \varphi_{1}^{P}}{\partial z^{2}} & =\left(u_{x}^{P}-1\right) \frac{\partial \varphi_{0}}{\partial x} \\
\frac{\partial^{2} \varphi_{1}^{B}}{\partial z^{2}} & =u_{x}^{B} \frac{\partial \varphi_{0}}{\partial x} .
\end{aligned}
$$

After calculation, one finds:

$$
\begin{aligned}
& \varphi_{1}(x, z, t)=\varphi_{1}^{P}(x, z, t)+\varphi_{1}^{B}(x, z, t) \\
& \varphi_{1}^{P}(x, z, t)=\frac{\partial \varphi_{0}}{\partial x}\left(z^{3}-\frac{z^{4}}{2}-\frac{z^{2}}{2}+\frac{1}{60}\right) \\
& \varphi_{1}^{B}(x, z, t)=-\frac{\operatorname{Ra}}{1440}\left(\frac{\partial \varphi_{0}}{\partial x}\right)^{2}\left(12 z^{5}-30 z^{4}+20 z^{3}-1\right) .
\end{aligned}
$$

This relation combined with the velocity profile given by Eq. A12 can now be used to calculate the dispersion term $<u_{x} \varphi_{1}>$ in Eq. (A9), leading to Eqs. 23, 24,.

\section{Appendix B: Fedorchenko and Chernov analytical solution in the diffusive regimes}

Fedorchenko and Chernov [17] derived the analytic solution of Eq. (43) with the initial and boundary conditions given by Eqs. 25. 27):

$$
\begin{gathered}
\varphi_{0}(x, t)=\sqrt{\frac{t}{\pi}} \exp \left(-\frac{(t-x)^{2}}{4 t}\right) \\
+\frac{1}{2}\left\{\exp (x)(1+x+t) \operatorname{erfc}\left(\frac{-(t+x)}{2 \sqrt{t}}\right)-\operatorname{erfc}\left(\frac{t-x}{2 \sqrt{t}}\right)\right\} .
\end{gathered}
$$

The concentration gradient at the interface, used in Sec. III C, simply follows from the spatial derivation of Eq. (B1) at $x=0$ :

$$
\begin{aligned}
\left(\frac{\partial \varphi_{0}}{\partial x}\right)_{x=0, t}=\sqrt{\frac{t}{\pi}} \exp & \left(-\frac{t}{4}\right) \\
& +\left(1+\frac{t}{2}\right) \operatorname{erfc}\left(-\frac{\sqrt{t}}{2}\right) .
\end{aligned}
$$

From Eq. B1, one can also calculate the extent of the diffusive layer using Eq. 29, see Eq. (44).

\section{Appendix C: Approximate solutions using the integral method in the dispersive regime $\mathrm{C2}$}

At long time scales, the temporal and diffusive terms in Eq. (31) are negligible in the regime $\mathrm{C} 2$, and concentration profiles obey the following partial differential equation in the growing diffusive layer:

$$
\frac{\partial \varphi_{0}}{\partial x} \simeq \frac{(\mathrm{PeRa})^{2}}{\alpha} \frac{\partial}{\partial x}\left(\frac{\partial \varphi_{0}}{\partial x}\right)^{3}
$$

We define $\psi=\left(\frac{\partial \varphi_{0}}{\partial x}\right)^{2}$, and Eq. $\mathrm{C} 1$ becomes:

$$
\left(\frac{3}{2} \frac{(\mathrm{RaPe})^{2}}{\alpha} \frac{\partial \psi}{\partial x}-1\right) \psi^{1 / 2} \simeq 0 .
$$

As the concentration gradient steadily increases, $\psi^{1 / 2} \neq 0$, and one has thus:

$$
\frac{\partial \psi}{\partial x} \simeq \frac{2}{3} \frac{\alpha}{(\mathrm{RaPe})^{2}}
$$

leading after integration to

$$
\varphi_{0}(x, t) \simeq \sqrt{\frac{8 \alpha}{27}} \frac{1}{\mathrm{PeRa}}(x+G(t))^{3 / 2}+F(t),
$$

where $F$ and $G$ are two functions to be defined. We postulate following the integral method [29] that the relation:

$$
\begin{aligned}
& \varphi_{0}(x, t)=0 \text { for } x<-G(t), \\
& \varphi_{0}(x, t)=\sqrt{\frac{8 \alpha}{27}} \frac{1}{\mathrm{PeRa}}(x+G(t))^{3 / 2}+F(t) \text { for } x<-G(t),
\end{aligned}
$$

is a good approximation of the solution providing that it verifies both the boundary condition Eq. 27) and the global solute conservation Eq. (21). which leads to $F \simeq-1$, and:

$$
G(t)=\left(\frac{5}{2} \sqrt{\frac{27}{8 \alpha}} \mathrm{PeRa} t\right)^{2 / 5} .
$$

As shown in Fig. 3 this relation approximates well the concentration profiles in the dispersive regime. From this approximation, we can finally calculate $\delta(t)$ using Eq. 29] leading to Eq. (45).

\section{Appendix D: Validity of the 1D advection dispersion model}

We consider the two cases defined in section IIID.

a. Case 1: $\mathrm{PeRa} / \sqrt{\alpha} \ll 1$. We aim at demonstrating that if $\mathrm{Pe} \lesssim 1$, then conditions Eqs. 51 53 hold for time $t \gg t_{\mathrm{D} 2 \rightarrow \mathrm{C} 2}$, where $t_{\mathrm{D} 2 \rightarrow \mathrm{C} 2}$ is given by Eq. (42). The condition Eq. (51) is satisfied because $t \gg t_{\mathrm{D} 2 \rightarrow \mathrm{C} 2} \gg 1$ and $\mathrm{Pe} \lesssim 1$. Similarly, the condition Eq. (52) is true because $t \gg t_{\mathrm{D} 2 \rightarrow \mathrm{C} 2}$ implies $\delta \gg 1$ (see scalings 40 41). Using Eq. A15 and assuming $1440 \sim \sqrt{\alpha}$, the condition Eq. 53 reads

$$
\operatorname{Pe} \frac{\operatorname{PeRa}}{\sqrt{\alpha}} \frac{\varphi_{0}}{\delta^{2}} \ll 1 .
$$

Using scalings 40,41, Eq. (D1) reduces to

$$
\operatorname{Pe}\left(\frac{\operatorname{PeRa}}{\sqrt{\alpha}} t\right)^{-1 / 5} \ll 1,
$$

this condition being obviously true for $t \gg t_{\mathrm{D} 2 \rightarrow \mathrm{C} 2}$. 
b. Case 2: PeRa $/ \sqrt{\alpha} \gg 1$. We aim at demonstrating that if condition Eq. (51) is true, then conditions Eqs. (52,53) are also true. $\delta \gg \mathrm{Pe}(\mathrm{PeRa} / \sqrt{\alpha})^{1 / 3} \gg \mathrm{Pe}$ in the $\mathrm{C} 1$ regime (from scaling 38) with $t \gg \mathrm{Pe}^{2}$ ), and $\delta \gg 1$ in the $\mathrm{C} 2$ regime (from scaling 40 41), which proves the validity of condition (52). In the $\mathrm{C} 1$ regime, condition (53) turns to Eq. (D1) and then to $\operatorname{Pe} t^{-1 / 2} \ll 1$ (using scaling 38 ), which is true for $t \gg \mathrm{Pe}^{2}$. In the $\mathrm{C} 2$ regime, condition (53) still leads to Eqs. D1 and (D2), the latter being true for $t \gg \mathrm{Pe}^{2}$.
[1] L. Daubersies, J. Leng, and J.-B. Salmon, "Confined drying of a complex fluid drop: phase diagram, activity, and mutual diffusion coefficient," Soft Matter 8, 5923 (2012).

[2] S. J. Lee, J. Hong, and Y.-S. Choi, "Evaporation-induced flows inside a confined droplet of diluted saline solution," Langmuir 30, 7710-7715 (2014).

[3] Tapan Kumar Pradhan and Pradipta Kumar Panigrahi, "Convection inside condensing and evaporating droplets of aqueous solution," Soft Matter 14, 4335 (2018).

[4] C. Loussert, A. Bouchaudy, and J.-B. Salmon, "Drying dynamics of a charged colloidal dispersion in a confined drop," Phys. Rev. Fluids 1, 084201 (2016).

[5] Yaxing Li, Christian Diddens, Pengyu Lv, Herman Wijshoff, Michel Versluis, and Detlef Lohse, "Gravitational Effect in Evaporating Binary Microdroplets," Phys. Rev. Lett. 122, 114501 (2019).

[6] Kwan Hyoung Kang, Hee Chang Lim, Hee Woong Lee, and Sang Joon Lee, "Evaporation-induced saline Rayleigh convection inside a colloidal droplet," Physics of Fluids 25, 042001 (2013).

[7] A. M. J. Edwards, P. S. Atkinson, C. S. Cheung, H. Liang, D. J. Fairhurst, and F. F. Ouali, "Density-Driven Flows in Evaporating Binary Liquid Droplets," Phys. Rev. Lett. 121, 184501 (2018).

[8] Yang Gu, Varun Hegde, and Kyle J.M. Bishop, "Measurement and mitigation of free convection in microfluidic gradient generators," Lab Chip 18, 3371 (2018).

[9] B. Selva, L. Daubersies, and J.-B. Salmon, "Solutal convection in confined geometries: enhancement of colloidal transport," Phys. Rev. Lett. 108, 198303 (2012).

[10] T. M. Squires and S. R. Quake, "Microfluidics: fluid physics at the nanoliter scale," Rev. Mod. Phys. 77, 977 (2005).

[11] E. R. Dufresne, D. J. Stark, N. A. Greenblatt, J. X. Cheng, J. W. Hutchinson, L. Mahadevan, and D. A. Weitz, "Dynamics of fracture in drying suspensions," Langmuir 22, 7144 (2006).

[12] S. Inasawa and Y. Yamaguchi, "Self-organized pattern formation of cracks perpendicular to the drying direction of a colloidal suspension," Soft Matter 8, 2416-2422 (2012).

[13] F. Boulogne, L. Pauchard, F. Giorgiutti-Dauphiné, R. Botet, R. Schweins, M. Sztucki, J. Li, B. Cabane, and L. Goehring, "Structural anisotropy of directionally dried colloids," Europhys. Lett. 105, 38005-38010 (2014).

[14] P. Lidon and J.-B. Salmon, "Dynamics of unidirectional drying of colloidal dispersions," Soft Matter 10, 4151-4161 (2014).

[15] K. Roger, M. Liebi, J. Heimdal, Q.D. Pham, and E. Spaar, "Controlling water evaporation through self-assembly," Proc. Natl. Acad. Sci. USA 113, 10275-10280 (2016).

[16] L. Goehring, J. Li, and P.-C. Kiatkirakajorn, "Drying paint: from micro-scale dynamics to mechanical instabilities," Philosophical Transactions of the Royal Society A: Mathematical, Physical and Engineering Sciences 375, 20160161 (2017).

[17] A. I. Fedorchenko and A. A. Chernov, "Exact solution of the problem of gas segregation in the process of crystallization,"
Int. J. Heat Mass Transfer 46, 915 (2003).

[18] Richard P. Sear and Patrick B. Warren, "Diffusiophoresis in nonadsorbing polymer solutions: The Asakura-Oosawa model and stratification in drying films," Phys. Rev. E 96, 062602 (2017).

[19] M. Emin Erdogan and P. C. Chatwin, "The effects of curvature and buoyancy on the laminar dispersion of solute in a horizontal tube," J. Fluid Mech. 29, 465 (1967).

[20] W. R. Young and Scott Jones, "Shear dispersion," Phys. Fluids A 3, 1087 (1991).

[21] J.S. Godfrey, "A numerical model of the james river estuary, virginia, u.s.a.” Estuarine Coastal Mar. Sci. 11, 295 (1980).

[22] Ronald Smith, "Longitudinal dispersion of a buoyant contaminant in a shallow channel," J. Fluid Mech. 78, 677 (1976).

[23] D. J. Maclean and T. Alboussière, "Measurement of solute diffusivities. part i. analysis of coupled solute buoyancy-driven convection and mass transport," Int. J. Heat Mass Transfer 44, 1639 (2001).

[24] M. L. Szulczewski and R. Juanes, "The evolution of miscible gravity currents in horizontal porous layers," J. Fluid Mech. 719, 82-96 (2013).

[25] A. Sarkar and M. S. Tirumkudulu, "Consolidation of charged colloids during drying," Langmuir 25, 4945 (2009).

[26] W. B. Russel, D. A. Saville, and W. R. Schowalter, Colloidal dispersions (Cambridge University Press, 1989).

[27] A. Oron, S.H. Davis, and S.G. Bankoff, "Long-scale evolution of thin liquid films," Rev. Mod. Phys. 69, 931 (1997).

[28] A. Bejan, Convection Heat Transfer, 2nd ed. (1995).

[29] M. N. Özisik, Heat conduction, edited by Inc. John Wiley \& Sons (1993).

[30] J.-B. Salmon, F. Doumenc, and B. Guerrier, "Humidityinsensitive water evaporation from molecular complex fluids," Phys. Rev. E 96, 032612 (2017).

[31] D. R. Vogus, V. Mansard, M. V. Rapp, and T. M. Squires, "Measuring concentration fields in microfluidic channels in situ with a fabry-perot interferometer," Lab Chip 15, 1689 (2015).

[32] Christine Peters, Ludger Wolff, Sandra Haase, Julia Thien, Thorsten Brands, Hans Jürgen Koß, and André Bardow, "Multicomponent diffusion coefficients from microfluidics using Raman microspectroscopy," Lab Chip 17, 2768 (2017).

[33] A. Bouchaudy, C. Loussert, and J. B. Salmon, "Steady microfluidic measurements of mutual diffusion coefficients of liquid binary mixtures," AIChE 64, 358 (2018).

[34] B. Abécassis, C. Cottin-Bizonne, C. Ybert, A. Ajdari, and L. Bocquet, "Osmotic manipulation of particles for microfluidic applications," New Journal of Physics 11, 075022 (2009).

[35] R. Nery-Azevedo, A. Banerjee, and T. M. Squires, "Diffusiophoresis in ionic surfactant gradients," Langmuir 33, 9694 (2017).

[36] S Shin, E Um, B Sabass, J T Ault, M Rahimi, P B Warren, and $\mathrm{H}$ A Stone, "Size-dependent control of colloid transport via solute gradients in dead-end channels," PNAS 113, 257 (2016). 
[37] A. F. Routh, "Drying of thin colloidal films," Rep. Prog. Phys. 76, 046603-046633 (2013).
[38] R. V. Birikh, "Thermocapillary convection in a horizontal layer of liquid,” J. Appl. Mech. Phys. 3, 69 (1966). 